\title{
\begin{tabular}{l|l} 
Mitraries & DSpace@MIT
\end{tabular}
}

\author{
MIT Open Access Articles
}

\section{SURFACE ALBEDO AND SPECTRAL VARIABILITY OF CERES}

The MIT Faculty has made this article openly available. Please share how this access benefits you. Your story matters.

Citation: Li (\#\#\#), Jian-Yang et al. "SURFACE ALBEDO AND SPECTRAL VARIABILITY OF CERES." The Astrophysical Journal 817.2 (2016): L22. (C) 2016 The American Astronomical Society

As Published: http://dx.doi.org/10.3847/2041-8205/817/2//22

Publisher: IOP Publishing

Persistent URL: http://hdl.handle.net/1721.1/108361

Version: Final published version: final published article, as it appeared in a journal, conference proceedings, or other formally published context

Terms of Use: Article is made available in accordance with the publisher's policy and may be subject to US copyright law. Please refer to the publisher's site for terms of use. 


\title{
SURFACE ALBEDO AND SPECTRAL VARIABILITY OF CERES
}

\author{
Jian-YANG Li (李荐扬 $)^{1}$, Vishnu RedDY ${ }^{1}$, Andreas Nathues ${ }^{2}$, Lucille Le Corre ${ }^{1}$, MattheW R. M. IzaWA ${ }^{3,4}$, \\ Edward A. Cloutis ${ }^{3}$, Mark V. Sykes ${ }^{1}$, Uri Carsenty ${ }^{5}$, Julie C. Castillo-Rogez ${ }^{6}$, Martin Hoffmann ${ }^{2}$, Ralf Jaumann ${ }^{5}$, \\ Katrin Krohn $^{5}$, Stefano Mottola ${ }^{5}$, Thomas H. Prettyman ${ }^{1}$, Michael Schaefer ${ }^{2}$, Paul Schenk ${ }^{7}$, Stefan E. Schröder ${ }^{5}$, \\ David A. Williams ${ }^{8}$, David E. Smith ${ }^{9}$, Maria T. Zuber ${ }^{10}$, Alexander S. Konopliv ${ }^{6}$, Ryan S. Park ${ }^{6}$, \\ Carol A. RaYMond ${ }^{6}$, and Christopher T. Russell ${ }^{11}$ \\ ${ }^{1}$ Planetary Science Institute, 1700 E. Ft. Lowell Road, Suite 106, Tucson, AZ 85719, USA \\ ${ }_{2}$ Max Planck Institute for Solar System Research, Göttingen, Germany \\ ${ }^{3}$ University of Winnipeg, Winnipeg, Manitoba, Canada \\ ${ }^{5}$ Rerman Aerospace Center (DLR), Institute of Planetary Research, Berlin, Germany \\ ${ }^{6}$ Jet Propulsion Laboratory, California Institute of Technology, Pasadena, CA 91109, USA \\ ${ }^{7}$ Lunar and Planetary Institute, Houston, TX 77058, USA \\ ${ }^{8}$ School of Earth and Space Exploration, Arizona State University, Tempe, AZ 85287, USA \\ ${ }^{9}$ Solar System Exploration Division, NASA Goddard Space Flight Center, Greenbelt, MD 20771, USA \\ ${ }^{10}$ Department of Earth, Atmospheric and Planetary Sciences, Massachusetts Institute of Technology, Cambridge, MA 02139, USA \\ ${ }^{11}$ Institute of Geophysics and Planetary Physics, University of California, Los Angeles, CA 90095, USA \\ Received 2015 December 2; accepted 2016 January 13; published 2016 January 29
}

\begin{abstract}
Previous observations suggested that Ceres has active, but possibly sporadic, water outgassing as well as possibly varying spectral characteristics over a timescale of months. We used all available data of Ceres collected in the past three decades from the ground and the Hubble Space Telescope, as well as the newly acquired images by the Dawn Framing Camera, to search for spectral and albedo variability on Ceres, on both a global scale and in local regions, particularly the bright spots inside the Occator crater, over timescales of a few months to decades. Our analysis has placed an upper limit on the possible temporal albedo variation on Ceres. Sporadic water vapor venting, or any possibly ongoing activity on Ceres, is not significant enough to change the albedo or the area of the bright features in the Occator crater by $>15 \%$, or the global albedo by $>3 \%$ over the various timescales that we searched. Recently reported spectral slope variations can be explained by changing Sun-Ceres-Earth geometry. The active area on Ceres is less than $1 \mathrm{~km}^{2}$, too small to cause global albedo and spectral variations detectable in our data. Impact ejecta due to impacting projectiles of tens of meters in size like those known to cause observable changes to the surface albedo on Asteroid Scheila cannot cause detectable albedo change on Ceres due to its relatively large size and strong gravity. The water vapor activity on Ceres is independent of Ceres' heliocentric distance, ruling out the possibility of the comet-like sublimation process as a possible mechanism driving the activity.
\end{abstract}

Key words: methods: observational - minor planets, asteroids: individual (1 Ceres) - space vehicles - techniques: image processing - techniques: photometric

\section{INTRODUCTION}

Observations of the dwarf planet Ceres by NASA's Dawn spacecraft have revealed a surface peppered with bright spots associated with impact craters (Nathues et al. 2015). The bright spots are prominent albedo features on the otherwise uniform surface of Ceres in albedo and color. The compositional analysis reveals that these bright spots are likely made up of a mixture of water ice, salts, and dark background material, suggesting a briny subsurface source. Bright spots within impact craters Dantu $(125 \mathrm{~km})$ and Occator $(92 \mathrm{~km})$ have been linked to intermittent and localized water vapor sources observed by the Herschel Space Observatory in 2011-2013 (Küppers et al. 2014), and are, therefore, possibly related to the sublimation activity. Recent ground-based observations of Ceres also suggested short-term global spectral variability that was attributed to the changing amount of water ice on the surface (Perna et al. 2015). These observations suggest a possibly changing surface caused by water outgassing on timescales of decades to months. We searched for evidence of surface albedo and spectral variations that might be evidence for ongoing activity by comparing various historical data sets and the most recent Dawn Framing Camera (FC) data collected during the approach to Ceres. Our search covered three different time frames: long-term in decades; mid-term in years, and short-term in months.

\section{ANALYSIS}

\subsection{Full-disk Spectral Variability}

The spectral data archived in the Planetary Data System Small Bodies Node from four epochs (Vilas et al. 1998; Bus \& Binzel 2003; Lazzaro et al. 2006), from Perna et al. (2015) in two epochs, and from recent ground-based observations (Reddy et al. 2015a) span nearly three decades, and are suitable for long-term spectral variability of Ceres (Figure 1(a)). The spectra of Ceres from various data sets all show a nearly linear shape between 0.53 and $0.85 \mu \mathrm{m}$, with increasing spectral slopes with solar phase angle and absorptions $<0.54 \mu \mathrm{m}$ and $>0.9 \mu \mathrm{m}$. Therefore, we fit linear slopes to all spectra between 0.54 and $0.85 \mu \mathrm{m}$ to quantify the spectral shape variation. Figure 1(b) suggests that the variations in slope are ascribable to variations in phase angle, except for the Dawn 
a)

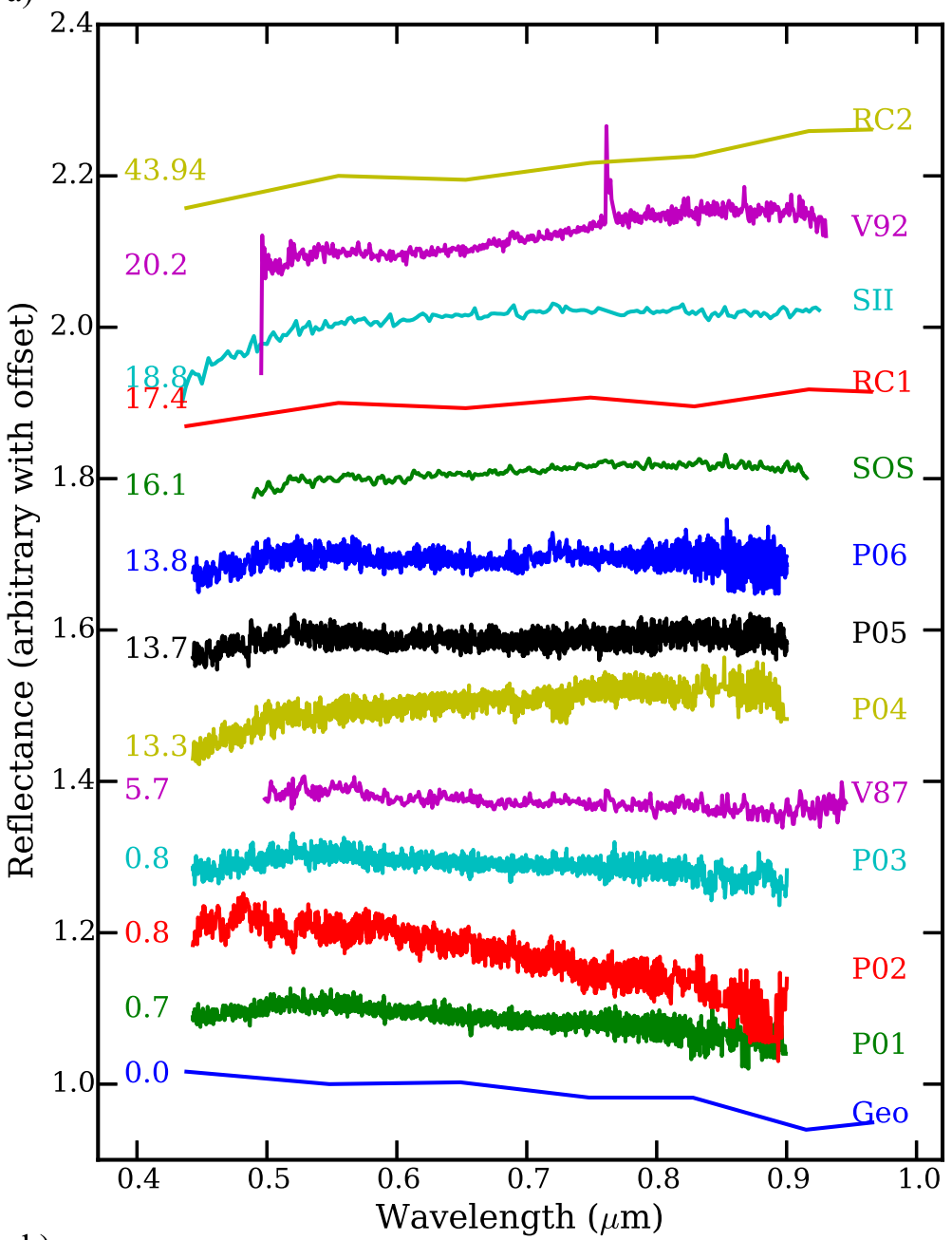

b)

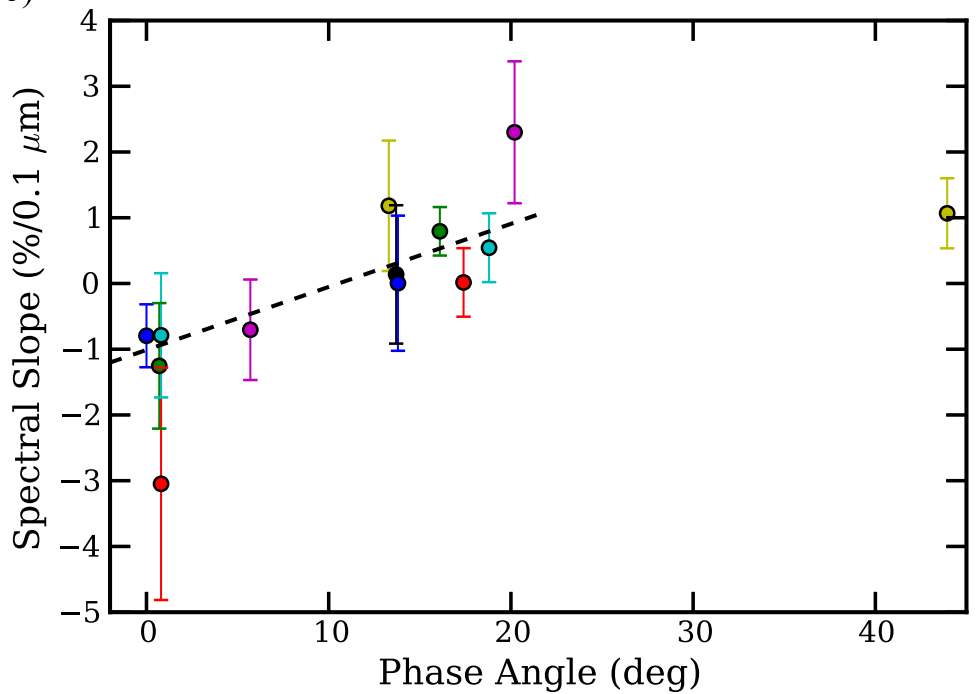

Figure 1. (a) The visible spectra of Ceres taken at increasing phase angles (marked at the left of each spectrum) from bottom to top (vertically shifted for clarity). From bottom to top: Geo: Modeled geometric albedo spectrum from ground-based photometric observations (Reddy et al. 2015a); P01, P02, P03: Perna et al. (2015) spectra on 2012 December 18 and 19; V87: Vilas et al. (1998) spectrum in 1987; P04, P05, P06: Perna et al. (2015) spectra on 2013 January 18-20; SOS: S ${ }^{2}$ OS $^{3}$ (Lazzaro et al. 2006); RC1: Dawn RC1; SII: SMASS II (Bus \& Binzel 2003); V92: Vilas et al. (1998) spectrum in 1992; and RC2: Dawn RC2. (b) The linear spectral slopes of Ceres' spectra between 0.54 and $0.85 \mu \mathrm{m}$. The colors of symbols correspond to the colors in panel (a). The large error bar for the red symbol at 0.8 phase angle, corresponding to the P02 spectrum, is due to the much higher noise in the spectrum compared to others. The black dashed line is a linear fit to the spectral slope with respect to phase angle, not including the slope measured from Dawn RC2 at a $44^{\circ}$ phase angle. The spectral slope of Ceres increases nearly linearly from a $0^{\circ}$ to $25^{\circ}$ phase angle. 
Table 1

Observation Conditions Extracted from the FC Color Data

\begin{tabular}{lcccc}
\hline \hline & $\begin{array}{c}\text { Phase } \\
\text { Angle } \\
(\text { degree })\end{array}$ & $\begin{array}{c}\text { Average Reso- } \\
\text { lution } \\
\left(\mathrm{km} \mathrm{pixel}^{-1}\right)\end{array}$ & $\begin{array}{c}\text { S/C Dis- } \\
\text { tance } \\
(\mathrm{km})\end{array}$ & $\begin{array}{c}\text { Heliocentric } \\
\text { Distance } \\
(\mathrm{AU})\end{array}$ \\
\hline $\mathrm{RC} 1$ & $17.2-17.6$ & 7.68 & 82,151 & 2.8529 \\
$\mathrm{RC} 2$ & $42.7-45.3$ & 4.24 & 45,359 & 2.8578 \\
RC3 (Equa- & $7.7-11.0$ & 1.28 & 13,603 & 2.9051 \\
$\quad$ & & & & \\
\hline
\end{tabular}

RC2 observations at a $44^{\circ}$ phase angle (described below), possibly indicating that the approximate linear relationship between spectral slope and phase angle stops somewhere between $20^{\circ}$ and $40^{\circ}$ phase angle.

Dawn FC observed Ceres for at least one full rotation of Ceres of $9.075 \mathrm{hr}$ (Chamberlain et al. 2007) in three separate epochs in 2015 February and April during the approach. These three observations are named Rotational Characterization (RC1, RC2, and RC3, see Table 1). The disk of Ceres did not fill the field of view of the FC during the RCs. Image calibration and spectral cube computation have been performed according to Reddy et al. (2012a) and Nathues et al. (2014). The time difference between $\mathrm{RC} 1$ and $\mathrm{RC} 2$ is one week and between RC2 and RC3 is 10 weeks, enabling us to explore short-term (days/weeks/months) global spectral variability on Ceres. To minimize dependence on the effect of the absolute calibration of the instrument, we calculated the relative spectra in each of the three RCs. The phase angle of RC3 is about $8^{\circ}$. Based on the linear fit to the spectral slope with respect to phase angle (Figure 1(b)), the spectral slope of Ceres at the RC3 phase angle is near zero. Therefore, the ratio spectra of $\mathrm{RC} 1$ and RC2 to RC3 should remove the effect of the uncertain absolute radiometric calibration of Dawn FC (Schröder et al. 2013a, 2014), and approximate the spectra of Ceres collected at $\mathrm{RC} 1\left(17^{\circ}\right.$ phase angle) and $\mathrm{RC} 2\left(44^{\circ}\right)$. The spectral slope from $\mathrm{RC} 1$ is consistent with the overall trend with phase angle in the three RCs. We therefore based our spectral slope analysis of Dawn FC data on these relative spectra (Figure 1(a)).

\subsection{Global Photometric Variability}

To measure global variations in the albedo of Ceres over a timescale of years, we used previously published Hubble Space Telescope (HST) data collected in 2003 and 2004 with the Advanced Camera for Survey (ACS) High Resolution Channel (HRC) through the broadband F555W filter (Li et al. 2006), supplemented by observations taken with the Wide Field Camera 3 (WFC3) UVIS channel through the broadband filter F555W in 2014 (GO-13503, PI: B.E. Schmidt). The WFC3/ UVIS F555W has a similar combined throughput as the ACS/ HRC F555W filter in 2014. We scaled the total flux of Ceres to a phase angle of $6^{\circ}$ by a linear fit to the disk-integrated phase function measured from the HST ACS/HRC data, which span a small range of phase angles from $5^{\circ} .4$ to $7^{\circ} .7$, and phasing each flux measurement to the rotation of Ceres based on the rotational period of Ceres, which has an accuracy of $7 \mathrm{~ms}$ per rotation (Chamberlain et al. 2007). The total reflected flux of Ceres measured through the three data sets agrees with each other to within $3 \%$, well within the absolute photometric calibration uncertainties of the two instruments.

\subsection{Occator Bright Spots}

The HST/ACS/HRC data and Dawn FC data are separated by 12 years, and are both spatially resolved, suitable for searching for albedo changes on local scales on Ceres in a medium time frame of years. We focus on the Occator crater because the bright spots inside this crater are undoubtedly the most prominent features on Ceres, and possibly related to the water vapor activity (Nathues et al. 2015). Comparison of absolute feature brightness between different data sets is extremely difficult, due primarily to the vastly different optical characteristics and absolute calibrations between HST and FC. Our approach is to compare the Occator bright spots with a reference region, for which we chose the Haulani crater $(32 \mathrm{~km}$ diameter), because it is relatively easy to identify in $H S T$ images. The underlying assumptions for our comparative approach is that the reflectances of Occator bright spots and the Haulani crater did not change simultaneously, and this is justified by the unchanging disk-integrated brightness of Ceres over the same time period (Section 2.2). We used RC1 images and RC3-equator images (Table 1) in this study. Note that the Haulani crater is in fact the brightest feature on Ceres in HST images (Thomas et al. 2005; Li et al. 2006), brighter than the Occator crater (Figure 2). The present study seeks an answer to the following question: Did the brightness of the Occator crater change since the previous HST observations, or are the different relative reflectance values of Haulani and Occator an observing artifact from comparing the HST and Dawn data sets?

\subsubsection{Photometric Modeling}

Precise photometric comparisons require corrections for different illumination and viewing geometries, which further rely on photometric models of the whole surface of Ceres and the bright spots. We separately modeled the photometric properties of Occator bright spots and the global average of Ceres based on the RC3 clear filter (effective wavelength $730 \mathrm{~nm}$, FWHM $370 \mathrm{~nm}$ ) data at $1.3 \mathrm{~km} \mathrm{pixel}^{-1}$ with the Hapke model (Hapke 2012), following the procedure outlined in Li et al. (2013). The shape model derived from Dawn FC data collected during approach is used, coupled with the spacecraft trajectory and pointing data in NASA's Navigation and Ancillary Information Facility SPICE kernels, to calculate the local illumination and scattering geometry. Some details of the photometric modeling are available in Li et al. (2015).

Due to the limited observing geometry available in our data, especially the lack of data at small phase angles to constrain the opposition effect, some modeled photometric parameters for Occator bright spots, such as the single-scattering albedo (SSA) and geometric albedo, highly depend on the assumptions in the modeling as well as the particular form of photometric models used. The Bond albedo, on the other hand, is much less modeldependent because it is dominated by the light scattered toward a moderate range of phase angles $\left(30^{\circ}-90^{\circ}\right)$ that is well sampled in the data.

Despite relatively large uncertainties in some modeled parameters, photometric models show that the bright spots have significantly different properties from the average Ceres. The SSA of the brightest part of Occator bright spots is between 0.67 and 0.80 , comparing to the average SSA of Ceres of 0.094-0.11. Occator bright spots have a Bond albedo of $0.24 \pm 0.01$ through the Dawn FC clear filter, comparing to 
a)

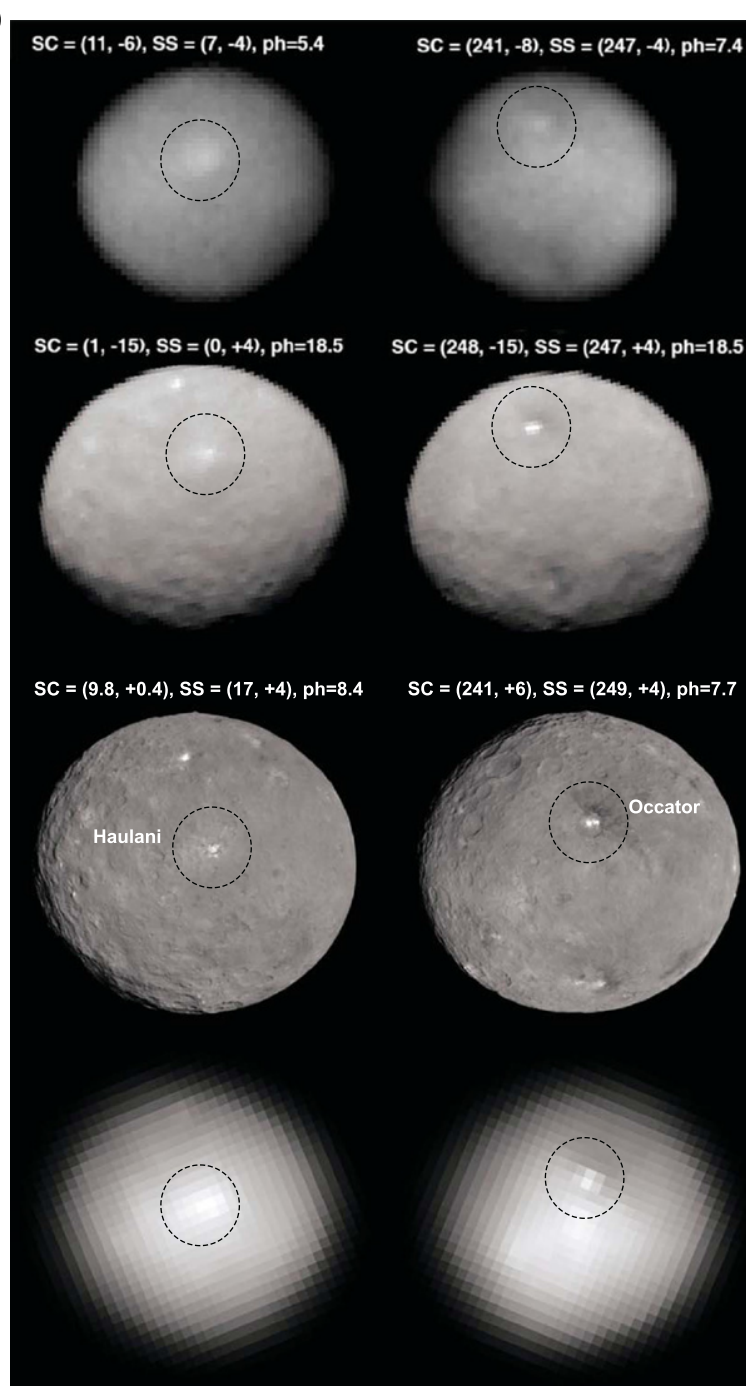

b)
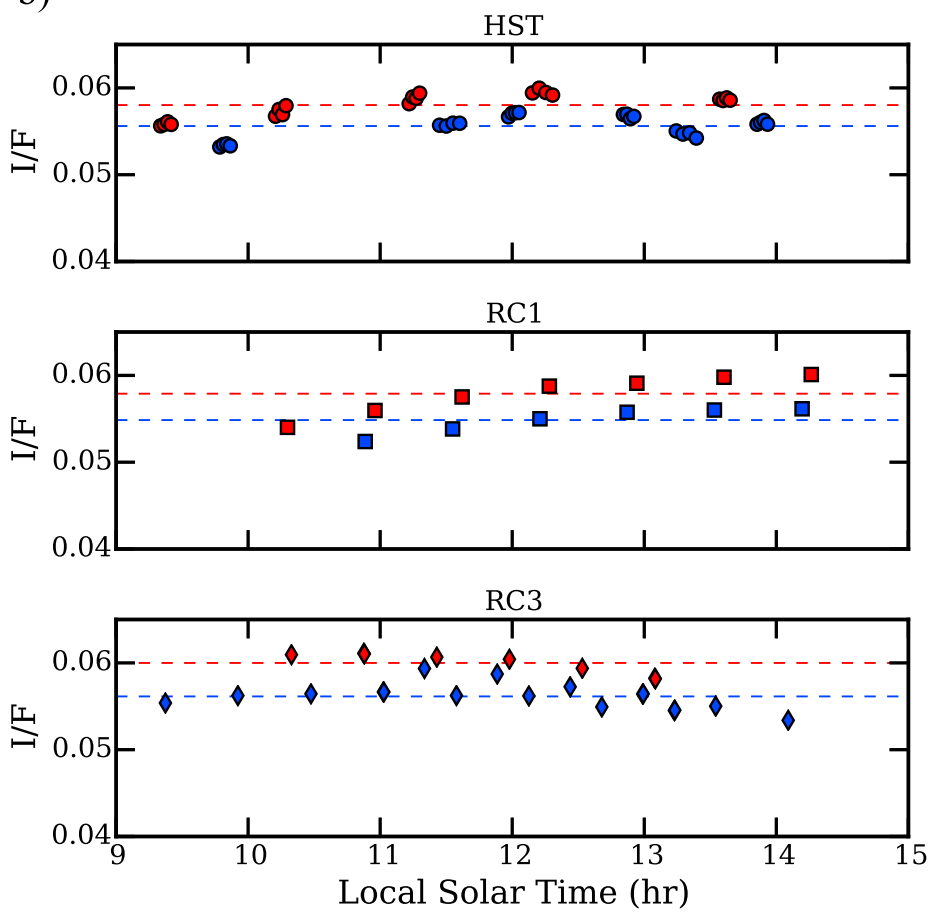

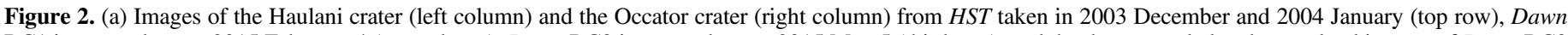

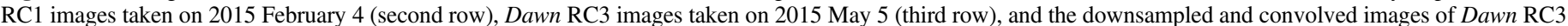

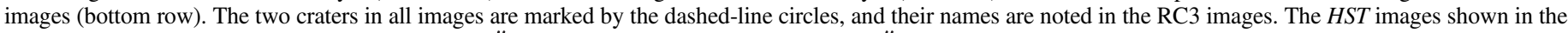

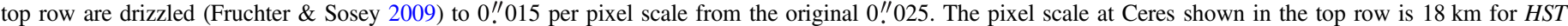

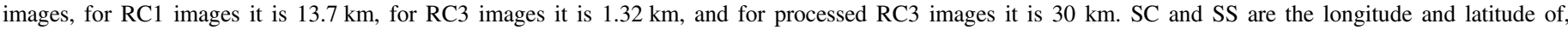

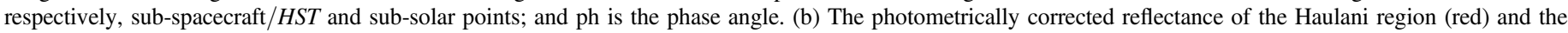

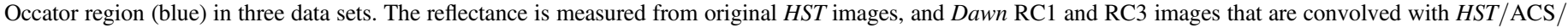

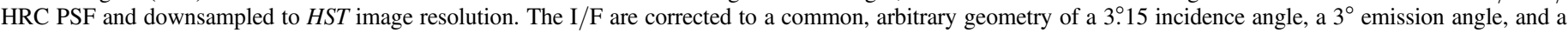

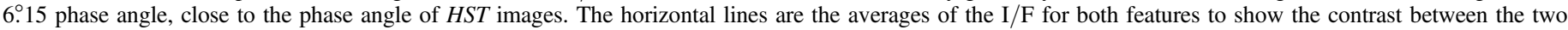

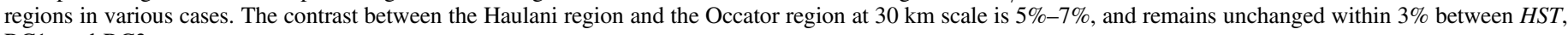
$\mathrm{RC} 1$, and RC3.

$0.034 \pm 0.001$ for the Ceres average. Since SSA and Bond albedo measure the scattered light toward all directions, they are more fundamental physical properties than the geometric albedo, which is often poorly constrained due to the lack of data at low phase angles. Hence, we compared Occator bright spots with other solar system objects in terms of their Bond albedos. The Bond albedo of Occator bright spots is higher than the average Vesta and most asteroids in the main belt, but is comparable to or slightly lower than the bright material on Vesta (Li et al. 2012; see our Figure 3). Compared to icy moons in the outer solar system, the Occator brightest spot is comparable or brighter than Ganymede and Callisto, but darker than Europa and most large Saturnian satellites whose surfaces are dominated by water ice. Therefore, Occator bright spots are unlikely pure water ice, but either admixtures of water ice, salts, or perhaps other bright minerals with darker background material.

The bright spots appear to scatter light more isotropically, with a single-term Henyey-Greenstein parameter of $-0.1 \pm 0.05$, than the average Ceres surface $(-0.35 \pm 0.05)$, which is consistent with most asteroids (C- and S-types). The phase function of a surface is affected by, among other factors, grain transparency, shape, defects, impurities, etc. The relatively less backscattering phase function of the bright spots is consistent with higher albedo and stronger multiple scattering, and possibly more transparent particles. The Hapke model roughness parameter for the bright spots is between $40^{\circ}$ and $50^{\circ}$, significantly higher than the average Ceres of $20^{\circ} \pm 3^{\circ}$. Relatively higher roughness suggests more loosely packed regolith particles that cast substantial shadows 


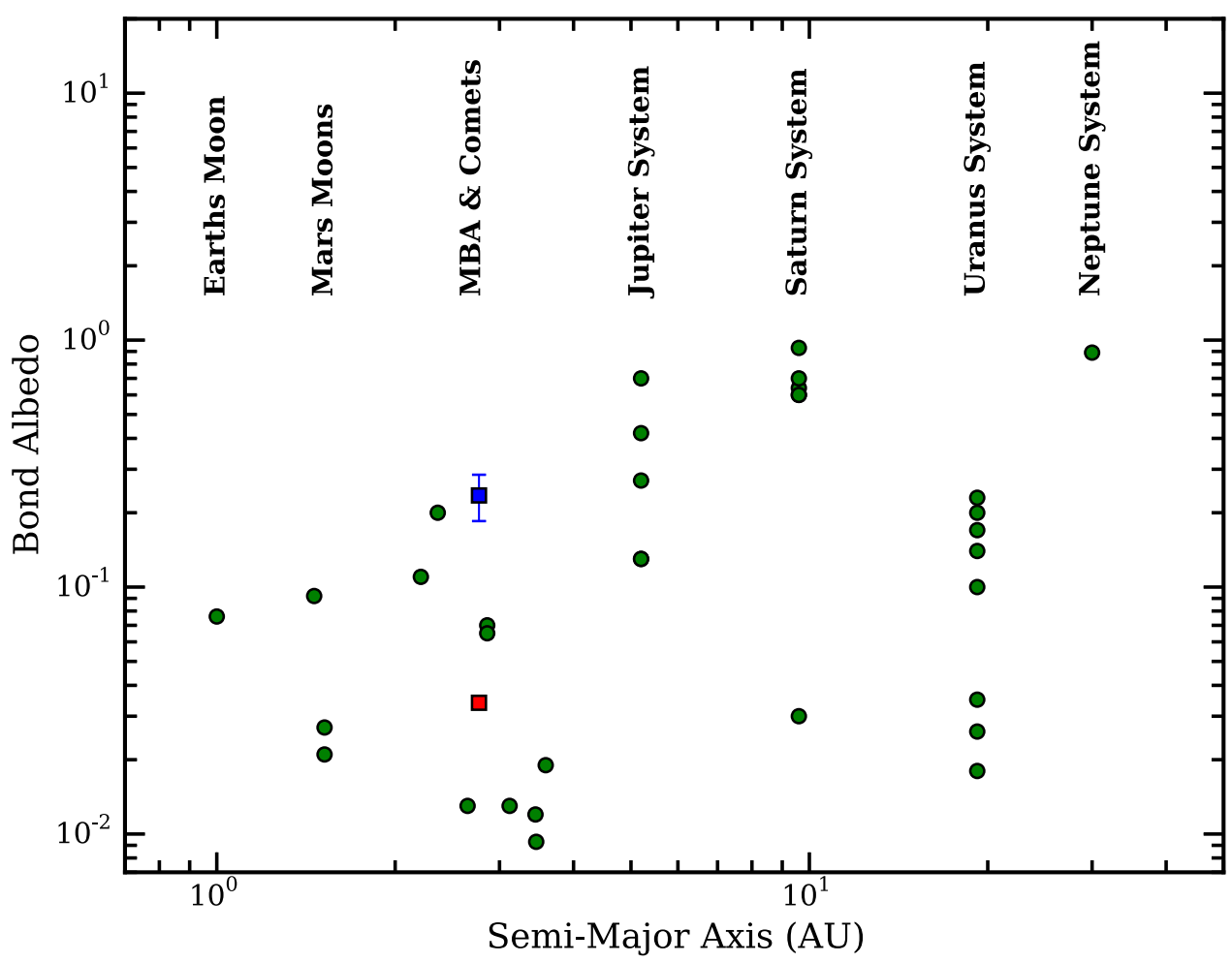

Figure 3. Comparisons of Bond albedos between Ceres (red square), Occator bright spots (blue square), and other solar system objects (green circles). The Bond albedos of icy satellites are from a list complied by Verbiscer et al. (2012); and those of comets (9P, 19P, 81P, and 103P), asteroids (asteroid numbers 4, 243, 253, 433, 951, 2867, and 26143), the moon, and the Martian satellites are from a list compiled by Li et al. (2013).

compared to the average Ceres, consistent with a geologically young regolith that has not been substantially processed (Schröder et al. 2013b).

\subsubsection{Albedo Variability of Occator Bright Spots}

For comparisons of the brightness between Occator bright spots and the Haulani crater in different data sets, the effects of different resolution and the vastly different point-spread function (PSF) have to be accounted for. From the radiometrically calibrated images of $\mathrm{RC} 1$ and $\mathrm{RC} 3$, first, we downsampled them to the pixel scale of HST images $\left(30 \mathrm{~km} \mathrm{pixel}^{-1}\right)$, then convolve the images with a PSF of HST ACS/HRC at F555W filter generated by the TinyTIM (Krist et al. 2011). We then measured the intensity value of the brightest pixel that contains the Occator crater and the Haulani crater from both HST images and the processed Dawn FC images. After correcting for the varying local illumination and viewing angles for both features in all measurements using the best-fit photometric parameters of the Ceres surface, we plot the brightness measurements with respect to local solar time for both features and compare their relative brightness (Figure 2(b)). The effect of subpixel shift in the downsampling process is negligible.

Ideally, after the correction for local illumination and viewing angle with the best-fit photometric model, the brightness of both features should be independent of local solar time. The residual variations with local solar time as shown in Figure 2(b) suggest that the photometric model does not completely remove the dependence on (varying) local scattering geometry over a Ceres day for both features. The slightly different illumination and observing geometries in different data sets result in different trends as they rotate over the disk of Ceres. However, note that the variations after correction in Figure 2(b) are at the 4\% level, within the performance expectation of photometric correction in general.

Figure 2(b) shows that the reflectance contrast between the Occator region and the Haulani region remains unchanged within $2 \%$ in HST, and Dawn FC RC1 and RC3 images. The reason that the Occator crater appeared to be darker than the Haulani region in HST images, as well as in the processed RC1 and RC3 images (Figure 2(b)), is due to the large footprint of $H S T$ pixels and the wide PSF (80\% energy encircled at 5 pixel; Maybhate et al. 2010) of the ACS/HRC camera, together washing out the high contrast of the relatively small bright spots $(<4 \mathrm{~km})$ with respect to the background. The bright Haulani crater region, together with the bright rays outside of the crater, are $>50 \mathrm{~km}$ across. Figure 2(b) also shows that no brightness variation was observed in Occator between the RC1 and RC3 observations over the span of 11 weeks. We will continue monitoring the brightness of the bright spots as well as all possible active regions on Ceres throughout the Dawn mission at resolutions down to $35 \mathrm{~m} \mathrm{pixel}^{-1}$ to search for any possible change.

\section{DISCUSSIONS}

To interpret the putative albedo variability on Ceres, we calculated the area in the Occator bright spots and how much albedo change is needed to create a measurable change in global albedo of Ceres. Our albedo variation investigation is limited by the $H S T$ pixel scale of $30 \mathrm{~km}$. The Occator bright spots have a combined area of about $95 \mathrm{~km}^{2}$, accounting for $11 \%$ of the $H S T$ pixel footprint. Therefore, our study suggests that any brightness change within the bright spots, or change in their total area, is smaller than $15 \%$. 


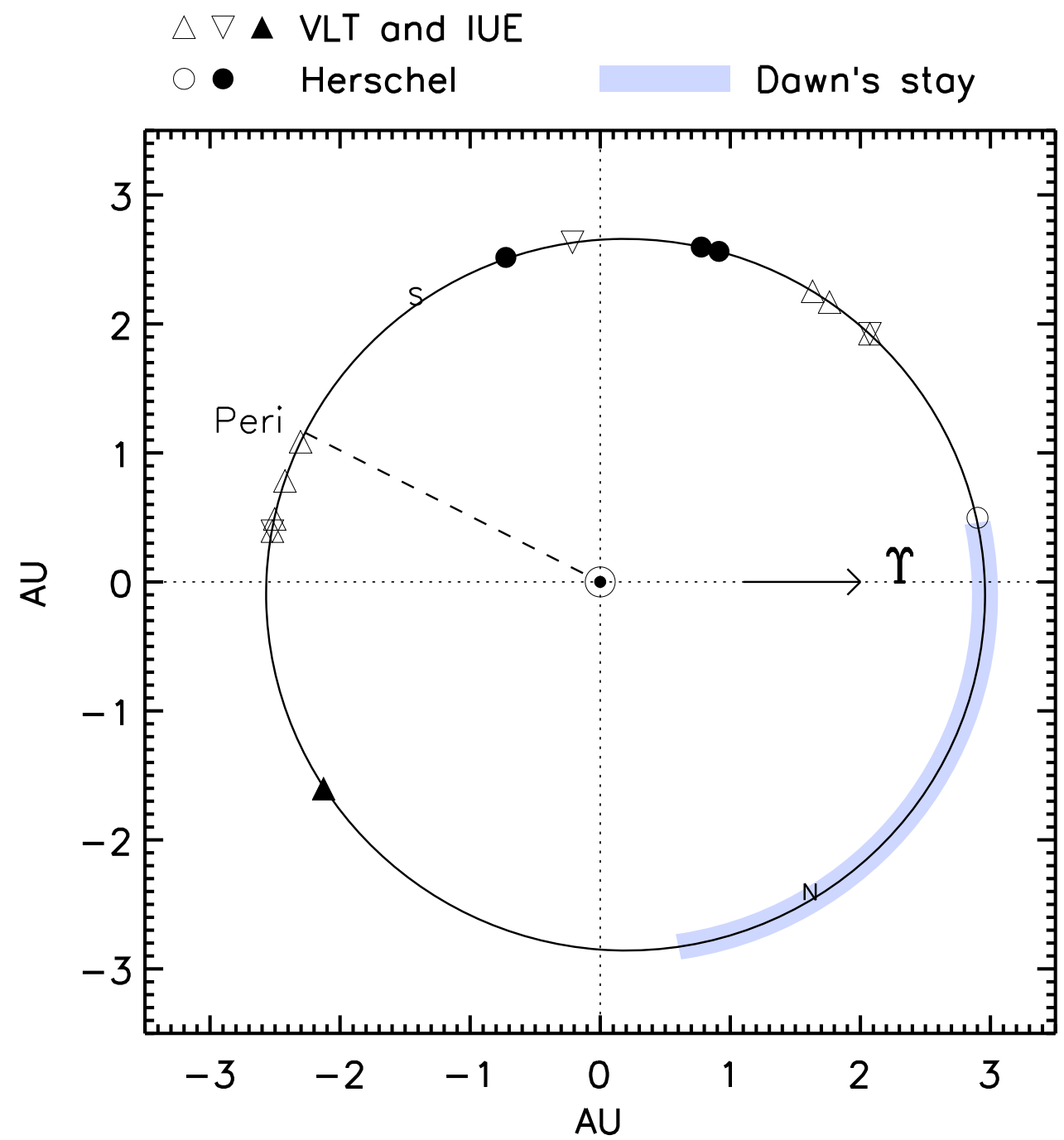

Figure 4. Positions of Ceres in its orbit during the previous searches of water vapor. The solid ellipse is Ceres' orbit with the Sun at the center, and "Peri" marks the perihelion of Ceres. The filled symbols mark the detection of water vapor (circles, by Herschel) or OH (triangles, by IUE), and the open symbols are non-detection. The directions of the triangles mark the direction of the off-limb $\mathrm{OH}$ search, where upper triangles are off the northern limb, and down triangles are off the southern limb. The $\mathrm{S}$ and $\mathrm{N}$ are summer solstice for southern hemisphere and northern hemisphere, respectively, based on the pole measurement from Dawn data. The blue shaded part of Ceres' orbit is during Dawn's planned stay from 2015 April 1 to 2016 June 30. No obvious trend of water outgassing and orbital phase (perihelion/ aphelion, seasons) is clearly identified. The outgassing appears to be intermittent and occurs at arbitrary orbital phases.

Given the water production rate of $2 \times 10^{26}$ molecules s $^{-1}$ for Ceres (Küppers et al. 2014), assuming a Bond albedo of 0.034 and a thermal emissivity of 0.9 , the pure water ice sublimation model (Cowan \& A'Hearn 1979) suggests an active area of $0.2 \mathrm{~km}^{2}$ for a slow rotator case where the surface is at instantaneous thermal equilibrium with local solar insolation at the latitude of Occator $\left(\sim 20^{\circ}\right)$. The low thermal inertia of Ceres of $<15 \mathrm{~J} \mathrm{~m}^{-2} \mathrm{~s}^{-0.5} \mathrm{~K}^{-1}$ (Chamberlain et al. 2009) justifies the slow rotator assumption. This active area corresponds to $0.2 \%$ of the combined surface area of the Occator crater spots, or $0.5 \%$ of the central core bright spot. The active area within the bright spots is too small to produce measurable albedo changes at $30 \mathrm{~km}$ pixel size.

The impact ejecta observed on main belt asteroid (596) Scheila during the impact in 2010 (Ishiguro et al. 2011) produced an average brightness change of $\sim 5 \%$ over a $10,000 \mathrm{~km}^{2}$ area. If we use the crater-size scaling law (Housen \& Schmidt 1983) with an exponent parameter of 0.7, based on an escape velocity of $55 \mathrm{~m} \mathrm{~s}^{-1}$ for Scheila (as calculated by Ishiguro et al. 2011) and $0.5 \mathrm{~km} \mathrm{~s}^{-1}$ for Ceres, the same impact on Ceres could produce an ejecta field that is $10^{4}$ smaller, or $\sim 1 \mathrm{~km}^{2}$. Scaling with albedo and size shows that such an impact is still too small to produce any albedo change on Ceres detectable by our analysis. Based on Bodewits et al.'s (2011) consideration, the projectile on Scheila had a size of 35-60 m and a relative velocity of $\sim 5.3 \mathrm{~km} \mathrm{~s}^{-1}$, and the impact frequency is once every $10^{3}$ years. Therefore, if the outgassing on Ceres were triggered by sporadic impact events similar to that considered for Scheila, then such events would not directly produce albedo changes with ejecta near the impact site detectable here.

Perna et al. (2015) suggested that Ceres displays short-term (months) visible spectral variability, and attributed it to "extended resurfacing processes such as cryovolcanism or cometary activity." Our interpretation of the ground-based visible wavelength spectral data shows an increasing spectral slope with increasing phase angle (Figure 1). Observation geometry has been shown to change slope and band depth in reflectance spectra of small bodies (Sanchez et al. 2012), including Vesta (Reddy et al. 2012b; Li et al. 2013). Hence our 
observations do not support the results reported in that study. The current intermittent and weak water sublimation does not cause albedo and color variations on the surface of Ceres detectable in our data.

Our analysis of the heliocentric dependence of activity on Ceres (Figure 4) shows no correlation between the two. This is an indication that whatever mechanism responsible for water vapor outgassing on Ceres is at least not completely driven by solar insolation as it is for comets. The presence of a network of cracks (Reddy et al. 2015b) inside craters with bright spots such as Occator hints at an internal source that is responsible for the transportation of volatiles from their subsurface sources.

We thank the Dawn operations team for the development, cruise, orbital insertion, and operations of the Dawn spacecraft at Ceres. The Framing Camera project is financially supported by the Max Planck Society and the German Space Agency, DLR. Li is supported by a subcontract from the University of California, Los Angeles under the NASA Contract \#NNM05AA86 Dawn Discovery Mission. This research made use of Astropy (Astropy Collaboration et al. 2013), and Matplotlib (Hunter 2007)

\section{REFERENCES}

Astropy Collaboration, Robitaille, T. P., Tollerud, E. J., et al. 2013, A\&A, 558, A33

Bodewits, D., Kelley, M. S., Li, J.-Y., et al. 2011, ApJL, 773, L3

Bus, S., \& Binzel, R. P. 2003, Small Main-belt Asteroid Spectroscopic Survey, Phase II, EAR-A-I0028-4-SBN0001/SMASSII-V1.0. NASA Planetary Data System

Chamberlain, M., Sykes, M. V., \& Esquerdo, G. A. 2007, Icar, 188, 451

Chamberlain, M. A., Lovell, A. J., \& Sykes, M. V. 2009, Icar, 202, 487
Cowan, J. J., \& A'Hearn, M. F. 1979, M\&P, 21, 155

Fruchter, A., \& Sosey, M. 2009, The Multidrizzle Handbook, Version 3.0 (Baltimore, MD: STScI)

Hapke, B. 2012, Theory of Reflectance and Emittance Spectroscopy (2nd ed.; Cambridge: Cambridge Univ. Press)

Housen, K. R., \& Schmidt, R. M. 1983, JGR, 88, 2485

Hunter, J. D. 2007, CSE, 9, 90

Ishiguro, M., Hanayama, H., Hasegawa, S., et al. 2011, ApJL, 740, L11

Krist, J. E., Hook, R. N., \& Stoehr, F. 2011, Proc. SPIE, 8127, 81270J

Küppers, M., O'Rourke, L., Bocklelée-Morvan, D., et al. 2014, Natur, 505, 525

Lazzaro, D., Angeli, C. A., Carvano, M., et al. 2006, Small Solar System Objects Spectroscopic Survey V1.0, EAR-A-I0052-8-S3OS2-V1.0. NASA Planetary Data System

Li, J.-Y., Le Corre, L., Schröder, S. E., et al. 2013, Icar, 226, 1252

Li, J.-Y., McFadden, L. A., Parker, J. Wm., et al. 2006, Icar, 182, 143

Li, J.-Y., Mittlefehldt, D. W., Pieters, C. M., et al. 2012, LPICo, 1667, 6386

Li, J.-Y., Nathues, A., Le Corre, L., et al. 2015, EPSC Abstracts, 10, EPSC2015-383

Maybhate, A., et al. 2010, ACS Instrument Handbook, Version 10.0 (Baltimore, MD: STScI)

Nathues, A., Hoffmann, M., Cloutis, E. A., et al. 2014, Icar, 239, 222

Nathues, A., Hoffmann, M., Schaefer, M., et al. 2015, Natur, 528, 237

Perna, D., Kan̆uchová, Z., Ieva, S., et al. 2015, A\&A, 575, L1

Reddy, V., Li, J.-Y., Gary, B. L., et al. 2015a, Icar, 260, 332

Reddy, V., Nathues, A., Le Corre, L., et al. 2012a, Sci, 336, 700

Reddy, V., Nathues, A., Le Corre, L., et al. 2015b, MetSoc, 2015, 5161

Reddy, V., Sanchez, J. A., Nathues, A., et al. 2012b, Icar, 217, 153

Sanchez, J. A., Reddy, V., Nathues, A., et al. 2012, Icar, 220, 36

Schröder, S. E., Maue, T., Gutiérrez Marqués, P., et al. 2013a, Icar, 226, 1304

Schröder, S. E., Mottola, S., Keller, H. U., Raymond, C. A., \& Russell, C. T. 2013b, P\&SS, 85, 198

Schröder, S. E., Mottola, S., Matz, K.-D., \& Roatsch, T. 2014, Icar, 234, 99

Thomas, P. C., Parker, J. Wm., McFadden, L. A., et al. 2005, Natur, 437, 224

Verbiscer, A. J., Helfenstein, P., \& Buratti, B. J. 2012, The Science of Solar System Ices, ed. M. S. Gudipati \& J. Castillo-Rogez (New York: Springer)

Vilas, F., Smith, B. A., McFadden, L. A., et al. 1998, Vilas Asteroid Spectra V1.1, EAR-A-3-RDR-VILAS-ASTEROID-SPECTRA-V1.1. NASA Planetary Data System 